\title{
Hepatic-Metabolite-Based Intermittent Fasting Enables a Sustained Reduction in Insulin Resistance in Type 2 Diabetes and Metabolic Syndrome
}

\section{(c) (i) (우 $\ominus$}

Authors

Markus Rohner ${ }^{1}$, Robert Heiz², Simon Feldhaus ${ }^{3}$, Stefan R. Bornstein ${ }^{4}$

\section{Affiliations}

1 EGB EpiGeneticBalance AG, Rheinfelden, Switzerland

2 Zentrum für Komplementärmedizin AG, Uster, Switzerland

3 Paramed AG, Haldenstrasse 1, Baar, Switzerland

4 Department of Medicine, Technische Universität Dresden, Dresden, Germany

Key words

insulin resistance, diabetes remission, non-alcoholic fatty liver, weight loss resistance, epigenetics

received 09.02.2021

accepted after revision 12.05.2021

published online 30.06 .2021

Bibliography

Horm Metab Res 2021; 53: 529-540

DOI 10.1055/a-1510-8896

ISSN 0018-5043

(c) 2021. The Author(s).

This is an open access article published by Thieme under the terms of the Creative Commons Attribution-NonDerivative-NonCommercial-License, permitting copying and reproduction so long as the original work is given appropriate credit. Contents may not be used for commercial purposes, or adapted, remixed, transformed or built upon. (https://creativecommons. org/licenses/by-nc-nd/4.0/)

Georg Thieme Verlag KG, Rüdigerstraße 14,

70469 Stuttgart, Germany

Correspondence

Prof. Stefan R. Bornstein

Director and Chair Department of Medicine

Dean of Development and International Affairs

Technische Universität Dresden

Fetscherstraße 74

01307 Dresden

Germany

Tel.: + 49351 4585955, Fax: + 493514586398

stefan.bornstein@uniklinikum-dresden.de

\section{ABSTRACT}

Insulin resistance is the hallmark of Type 2 Diabetes and is still an unmet medical need. Insulin resistance lies at the crossroads of non-alcoholic fatty liver disease, obesity, weight loss and exercise resistance, heart disease, stroke, depression, and brain health. Insulin resistance is purely nutrition related, with a typical molecular disease food intake pattern. The insulin resistant state is accessible by TyG as the appropriate surrogate marker, which is found to lead the personalized molecular hepatic nutrition system for highly efficient insulin resistance remission. Treating insulin resistance with a molecular nutrition-centered approach shifts the treatment paradigm of Type 2 Diabetes from management to cure. This allows remission within five months, with a high efficiency rate of $85 \%$. With molecular intermittent fasting a very efficient treatment for prediabetes and metabolic syndrome is possible, improving the non-alcoholic fatty liver disease (NAFL) state and enabling the body to lose weight in a sustainable manner. 


\section{Introduction}

Today, treatment of Type 2 Diabetes is glucose-centered and symptom-oriented. The history of most patients with Type 2 Diabetes is a gradual rise in blood glucose concentrations over time, despite medication, as reported by Madsen et al. [1]. Insulin resistance is the hallmark of Type 2 Diabetes, but is still an unmet medical need even though advances in diabetes medicines have been made over the last several years. Ferrannini et al. [2] reported that the same is true for the fast and simple detection of insulin resistance. Insulin resistance lies at the key crossroads of metabolic and mitochondrial dysfunction, visceral fat, non-alcoholic fatty liver (NAFL), muscle exercise and weight loss resistance, subclinical inflammation, metabolic syndrome (MetS), overweight, and obesity, and is characterized by epigenetically driven accelerated aging, as Nannini et al. [3] reported. Insulin resistance plays a key role in a reduced capability of the immune system to kill pathogens such as viruses and bacteria, as De Rosa et al. [4] and Chávez-Reyes et al. [5] reported. Vestergaard Jensen et al. [6] reported insulin resistance as an important risk factor for community-acquired pneumonia. Insulin resistance predisposes to cardiovascular disease and shortens human lifespan, as data from Gardner et al. [7] showed. Insulin resistance is reported to drive other major non-communicable diseases, such as high blood pressure, chronic kidney disease (Chen et al. [8]), depression (Won Lee et al. [9]; Geraets et al. [10], Ford et al. [12]), and stroke (Rundek et al. [11]). Alzheimer's disease and dementia are also highly correlated to increased levels of triglycerides at midlife as data from Nägga et al. [13] showed. Insulin resistance also underlies many obesity-related cancer developments, as reported by Sung et al. [14].

Insulin resistance is independently correlated with increased risk of incident diabetes in Chinese adults, as reported by Li et al. [15], and is closely related with higher mortality for COVID-19, according to data reported by Ren et al. [16]. Diabetes is the most important cause of mortality in COVID-19 hospitalized patients, as reported by Corona et al. [17]. A large-scale analysis reported by McGovern et al. [18] found that the COVID-19 mortality risk increases in patients with Type 2 Diabetes due to drastic biological age acceleration. The prevalence of Type 2 Diabetes in Switzerland is estimated to be between 5.7 and $7.0 \%$. Two-thirds of patients with Type 2 Diabetes are aware of their status, and over three-quarters of those who are aware are treated, according to data from Kaiser et al. [19]. In the US, adults with optimal metabolic health are a small percentage of the population: $17.6 \%$, according to Araujo et al. [20].

Type 2 Diabetes is observed to correlate with obesity regardless of the genetic risks. The currently available obesity-oriented treatment options for Type 2 Diabetes are either a bariatric operation, or a caloric weight-loss strategy that involves a very low-calorie diet, as reported by Kerr et al. [21], Willi et al. [22], and Xin et al. [23]. As observed by Baskota et al. [24], a bariatric operation can only be proposed for those with a $\mathrm{BMI}>30$ and should not be proposes for non-diabetic obese patients.

\section{$\mathrm{BMI}$ is not the main driver for insulin resistance; insulin resistance drives BMI}

Diabetes primarily depends on visceral fat and not BMI, according to data from Vistisen et al. [25]. Her group found that the great ma- jority of patients who had modest weight gains before diagnosis had the highest diabetes risk. Wu et al. [26] reported that, in Mexican Americans, metabolic health has a greater impact on diabetes than overweight/obesity. Eckel et al. [27] reported that the quality of metabolic health was decisive for diabetes progression, irrespective of BMI. Acquired obesity independent of genetic risk is primarily related to deleterious alterations in the lipid metabolism, per data found by Pitiläinen et al. [28] in studies of twins. Insulin resistance discriminates between healthy and unhealthy phenotypes of obesity and leanness, as reported by Owei et al. [29] and Mongraw-Chaffin et al. [30] and drives the unhealthy obese phenotype into developing Type 2 Diabetes.

Insulin resistance has its root not only in disturbed glucose metabolism but also in a derailed fatty acid metabolism leading to glucolipotoxic conditions and beta-cell death, as reported by Bagnati et al. [31]. Increased blood triglycerides accompanied by lower HDL values represent an insulin resistant metabolic status and are purely nutrition-related. Triglyceride levels showed a significant negative correlation with BMI and body fat. HDL cholesterol was significantly negatively correlated with waist circumference and positively correlated with body fat, as data from Telles et al. [32] showed.

Lipid indices, which can be easily calculated with routine laboratory tests, may be useful markers for insulin resistance risk assessments in clinical settings, according to Lee et al. [33]. The triglyceride to HDL ratio allow identification of the insulin-resistant metabolic state, and a high TG:HDL-C ratio at baseline may be a useful surrogate indicator of future Type 2 Diabetes, as reported by Lim et al. [34]. The TyG index, calculated based on fasting triglyceride and glucose blood values, correlates highly with insulin resistance. Both markers, the TG:HDL-C and TyG present a significant association with lifetime cardiovascular risks in adults reported by Rojas-Humpire et al. [35] and Si et al. [36]. The TyG index correlated highly with the epigenetic age acceleration, as data from Arpon et al. [37] showed. HOMA-IR had a significant positive correlation with the TyG and TG:HDL indices, as reported by Çin et al. [38] and Kron et al. [39].

Triglycerides are endogenously formed after an excess of glucose ingestion via a de novo lipogenesis pathway forming malonyl-CoA, diacylglycerols, and palmitic acid (16:0), and directly influence insulin resistance, as Lyu et al. [40] reported. Lee [41] reported palmitic acid 16:0 was positively associated with incident heart failure in older adults. Malonyl-CoA is a master regulator for insulin sensitivity. Hyperglycemia with hyperinsulinemia increases malonyl-CoA production and inhibits functional CPT1 activity, while and shunting long-chain fatty acids away from oxidation and towards storage in human muscle, as reported by Rasmussen et al. [42]. Malonyl-CoA is the first reaction step for the de novo lipogenesis of palmitic acid, which is positively associated with Type 2 Diabetes, as data from Imamura et al. [43] showed. But de novo lipogenesis is also positively associated with saturated fatty acid intake and is elevated in patients with non-alcoholic fatty liver and Type $2 \mathrm{Di}$ abetes, as reported by Imamura et al. [43]. The TyG index is significantly associated with incident NAFL, as reported by Kitae et al. [44] and jimenez-Rivera et al. [45]. Kolb et al. [46] reported detrimental consequences of prolonged high insulin concentrations and argues for a lifestyle that limits circadian insulin levels. Skipping 
breakfast a few times a week was associated with general adiposity and with general and central adiposity, as reported by Wadolowska et al. [47], and was also associated with a higher risk of insulin resistance, as data from Joo et al. [48] showed. Consuming a three-meal diet with a carbohydrate-rich breakfast allows insulin dose reduction, leading to weight loss and better glycemic control compared with an isocaloric six-meal diet, as reported by Jakubowicz et al. [49].

\section{Food choices for a molecular dietary pattern developing an insulin-resistant metabolic state}

Compared to normal glycemic adults, adults with Type 2 Diabetes make different food choices, favoring a high saturated fat diet with higher total fat and protein and less fiber, as reported by Breen et al. [50]. Increased insulin resistance favors continued unhealthy food choices via aberrant central insulin action, as reported by Tiedemann et al. [51], leading to a vicious cycle in which insulin resistance drives changes in taste perception, as observed by Pugnaloni et al. [52], leading to continued eating and snacking behavior. Saturated fat increases intramuscular triglycerides, and a decreased intake of saturated fatty acids could be beneficial in reducing intramuscular triglycerides and the associated risk of diabetes, according to data from Luukkonen et al. [53]. In obese subjects with normal glycemia, elevated circulating levels of free fatty acids during fasting is the major metabolic derangement candidate driving fasting hyperinsulinemia as a homeostatic response, as reported by Fryk et al. [54].

Maintaining glycemic control via a healthy fatty acid metabolism may be an emerging key factor for maintaining a healthy beta-cell formation for a normal glucagon metabolism, as reported by Grubelnik et al. [55]. Lower fiber intake of all types is associated with higher insulin levels. Triglyceride concentrations are potentially sensitive to fiber consumption, as reported by Hannon et al. [56]. Fiber intake at recommended levels may be associated with significant cardiometabolic benefits, as data from Dong et al. [57] showed. Higher intakes of dietary choline and betaine are associated with lower insulin resistance in the general population, as reported by Gao et al. [58].

The amount of dietary intake of animal protein was positively related to HOMA-IR, while plant protein was not significantly related to insulin resistance, as reported by Azemati et al. [59]. Plantbased proteins with polyphenols are reported by Meir et al. [60] and Castro-Barquero et al. [61] to improve HDL cholesterol and to have an inverse relationship with triglycerides. McKaye et al. [62] reported that a low intake of vitamin $D$, folate, magnesium, and potassium have a negative relationship to BMI, while other micronutrients, such as vitamins $\mathrm{B} 12$ and $\mathrm{E}$, do not.

\section{A personalized molecular feeding and control strategy for the remission of insulin resistance}

The molecular dietary pattern of humans is directly related to transcriptions generating epigenetically the enzymatic pattern needed for a healthy metabolism for maintaining energy homeostasis. An unhealthy dietary pattern leads via the epigenetically driven transcription to an unfavorable enzymatic pattern, leading to insulin resistance to rescue the energy homeostasis, as reported by Hall et al. [63].
The CPT1 gene expression is epigenetically silenced in the unhealthy obese phenotype, as data from Maples et al. [64] show. The $\beta$-oxidation genes are also downregulated during weight loss, preserving metabolic inflexibility. Metabolic flexibility is lacking at the extreme of the metabolic phenotype in obese youth with dysglycemia related to a defect in insulin sensitivity, limiting substrate utilization, as reported by Bacha et al. [65]. An epigenomic study by Irvin et al. [66] and Mamtani et al. [67] found that the CPT1 locus influenced by methylation is strongly and robustly associated with low-density lipoprotein cholesterol, triglycerides, as well as visceral fat and waist circumference. Lai et al. [68] found that the proportion of total energy supplied by carbohydrates and fat can have a causal effect on the risk of metabolic diseases, via the epigenetic status of the CPT1A gene directly influencing metabolic health through CPT1A gene activation or silencing. Epigenetic signatures are associated with dyslipidemia and are strongly associated with HOMA-IR, as a direct measure for insulin resistance. HOMA-IR values $\leq 3$ as the threshold for Type 2 Diabetes showed a different methylation pattern than for individuals with $\mathrm{HOMA}-\mathrm{IR}>3$, as reported by Arpon et al. [69].

Below we present a precisely designed and personalized molecular feeding strategy, integrating food research findings, and combining an epigenetic view aimed at the TyG and TG:HDL indices, for simple metabolic steering and control, for efficient insulin resistance normalization.

\section{Compliance with ethical standards}

Ethical approval: All patients were treated following an ethical approach to select the optimal treatment procedure according to their medical needs and the patients agreed before therapy began to possible reduction of their medications, when necessary.

Informed consent: Patient data and information have been completely anonymized.

\section{Materials and Methods}

\section{A personalized nutritional method for insulin resistance and diabetes remission}

Hepatic intermittent fasting therapy consists of two parts, always with a three meal per day diet. The first part is a very low calorie diet (VLCD) to reverse Type 2 Diabetes. The VLCD is followed by a personalized, hepatic-focused whole food diet, epigenetically oriented to stabilizing the metabolism that was reprogrammed in the VLCD part. Both parts, VLCD followed by the whole food diet, are used for diabetes remission therapy. The VLCD combines protein shakes (Protiline) with selected vegetable intake focused mainly on sulforaphane, betaine, and choline. The personalized, hepatic-focused whole food diet is applied for the normalization of MetS and prediabetes. The whole food diet steers and controls the personalized food intake, and is digitally supported on a molecular level for the individual threshold values of selected nutrients, calculated not just for the key influencing macro nutrition molecules of saturated fats, glucose, fiber, and proteins, but also for selected micronutrients, geared to achieve optimal TyG and TG:HDL values, according to Rohner [70]. 
The key molecules related to insulin resistance of the food are digitally calculated for molecular control. The healthy range of molecular concentration of the key food ingredients is estimated and visualized for the client for easy self-control, using the "EPIKonzept App." The client can simply follow daily his food selection and is informed about how well he is achieving on a molecular level the targeted biomarkers. Possible deviations are monitored and a buddy support system is put in place, for increasing the self-efficacy for continuous optimization.

\section{Supporting product formulas combined with therapy}

Both parts of the therapy, VLCD and a personalized whole food diet, are supported with tailored biochemically active product formulas, namely EPIGENOSAN and METHYLOSAN.

Epigenosan is a tableted formula product consisting of mate tea extract, oil of the microalgae schizochytrium, green tea extract, an isoflavone from soja extract, I-arginine, magnesium, niacin, pantothenic acid, folic acid, biotin, and vitamins D, E, B6, and B12.

The supplement's key ingredients are combined with l-carnitine I-tartrate, assuring a cofactor for the CPT1 enzyme complex, and is intermittently fed under fasting conditions. I-Carnitine supports energy metabolism and counteracts metabolic inflexibility, synchronizing the intermittent fasting method and supporting the functionality of the $\beta$-oxidation to accelerate the clearance of triglycerides. The product formula epigenosan is embedded into the diet, according to Rohner [71].

Epimethylosan is a capsuled formula product based on broccoli sprouts and white asparagus and includes choline, magnesium, I-methionine, coenzyme Q10, zinc, vitamins B2, B6 and B12, manganese, chrome, and folic acid mediating the one-carbon metabolism and avoiding undernutrition of key molecules for optimal redox reactions. Epimethylosan is always used together with epigenosan.

\section{Analytics applied and calculation of medical indices}

Commonly used analytical methods were applied to determine the measured parameters in the figures in the result section. The TyG index was estimated according to the formula Ln [fasting triglycerides $(\mathrm{mg} / \mathrm{dl}) \times$ fasting glucose $(\mathrm{mg} / \mathrm{dl}) / 2$ ], as published by Simental-Mendia et al. [72]. The TG:HDL ratio was obtained by dividing the triglyceride level $(\mathrm{mg} / \mathrm{dl})$ by the HDL-C level ( $\mathrm{mg} / \mathrm{dl}$ ) according to Masson et al. [73].

For the nutritional analysis, the DGExpert program was used.

The liver index was calculated according to the formula liver index:

$\left(\mathrm{e}^{0.953^{*} \text { loge (triglycerides) }+0.139 * \mathrm{BMI}+0.718^{*} \text { loge }(\mathrm{ggt})+0.053 * \text { waist }}\right.$

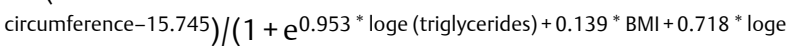
$(g g t)+0.053^{*}$ waist circumference -15.745$) \times 100$

according to Bedogni et al. [74].

\section{Diabetes remission group}

The Type 2 Diabetes group consisted of 13 patients ( $\vee$ Table 1 ). The inclusion criteria for the diabetes remission group were to be not older than 70 years old; diagnosed with Type 2 Diabetes for not longer than 7 years; no heart attack history; no depressive disorders at the time of application; no insulin treatment; and $\mathrm{BMI} \geq 25$. Diabetes remission was defined as glycated haemoglobin $\left(\mathrm{HbA}_{1 \mathrm{c}}\right)$ of less than $47.5 \mathrm{mmol} / \mathrm{mol}(<6.5 \%$ ) and fasting glucose of less than $7.0 \mathrm{mmol} / \mathrm{l}$ after at least 2 month off all antidiabetic medications.

\section{MetS/prediabetic therapy group}

The MetS group consisted of 21 patients ( $\triangleright$ Table 2 ). The inclusion criteria for the MetS/prediabetic group were no depressive disorders at the time of application; and $B M I \geq 20 \mathrm{~kg} / \mathrm{m}^{2}$.

- Table 1 Diabetes remission group participants overview.

\begin{tabular}{|c|c|c|c|c|c|c|c|}
\hline Patient & BMI initially & $\begin{array}{l}\text { BMI after } \\
60 \mathrm{~d}\end{array}$ & $\begin{array}{l}\text { BMI after } \\
150 \mathrm{~d}\end{array}$ & Sex & Age & $\begin{array}{l}\text { Antidiabetic } \\
\text { medication }\end{array}$ & Years diagnosed \\
\hline Diab 1 & 33.3 & 29.2 & 29.2 & $\mathrm{~F}$ & 55 & Metfin & 5 \\
\hline Diab 2 & 34.3 & 31.2 & 25.9 & $\mathrm{~F}$ & 62 & Metformin & 1 \\
\hline Diab 3 & 26.8 & 22.2 & 21.3 & M & 54 & Glavumet & 2 \\
\hline Diab 4 & 32.8 & 27.3 & 26.1 & $M$ & 55 & None & $<1$ \\
\hline Diab 5 & 31.4 & 26.5 & 24.6 & $\mathrm{~F}$ & 56 & Metfin & 7 \\
\hline Diab 6 & 31.5 & 27.1 & 25.2 & M & 47 & Metfin & 3 \\
\hline Diab 7 & 35.9 & 29.4 & 26.7 & M & 46 & Metfin & 4 \\
\hline Diab 8 & 26.8 & 24.5 & 22.6 & $\mathrm{~F}$ & 47 & None & $<1$ \\
\hline Diab 9 & 26.8 & 24.4 & 22.0 & M & 64 & Metfin & Unknown \\
\hline Diab 10 & 33.3 & 26.5 & 26.7 & M & 61 & None & Unknown \\
\hline Diab 11 & 54.3 & 47.6 & 46.5 & M & 50 & Janumet & 2 \\
\hline Diab 12 & 38.9 & 36.0 & 29.7 & $\mathrm{~F}$ & 68 & Januvia & 2 \\
\hline Diab 13 & 41.8 & 36.2 & 31.1 & $\mathrm{~F}$ & 35 & None & $<1$ \\
\hline Mean BMI & 34.4 & 29.9 & 27.5 & & & & \\
\hline
\end{tabular}


- Table 2 MetS/prediabetic group participant overview.

\begin{tabular}{|c|c|c|c|c|c|c|}
\hline Patient & Age & Sex & Medication & BMI initially & $\begin{array}{l}\text { BMI after Therapy } \\
\text { (150 d) }\end{array}$ & Initial metabolic status \\
\hline MetS 1 & 47 & w & None & 33.2 & 29.3 & Severe risk for insulin resistance \\
\hline MetS 2 & 60 & $\mathrm{~m}$ & None & 37.2 & 32.5 & Severe risk for insulin resistance \\
\hline MetS 3 & 49 & w & Antiepileptics & 31.9 & 30.7 & Severe risk for insulin resistance \\
\hline MetS 4 & 70 & w & None & 31.4 & 26.1 & Insulin resistant \\
\hline MetS 5 & 71 & $\mathrm{~m}$ & None & 26.5 & 22.9 & Insulin resistant \\
\hline MetS 6 & 84 & w & None & 27.8 & 26.2 & Prediabetic \\
\hline MetS 7 & 82 & w & Statin & 39.6 & 36.1 & Prediabetic \\
\hline MetS 8 & 51 & w & None & 23.0 & 20.7 & Insulin resistant \\
\hline MetS 9 & 53 & $\mathrm{~m}$ & None & 33.6 & 29.1 & Prediabetic \\
\hline MetS 10 & 32 & w & None & 24.6 & 21.3 & Insulin resistant \\
\hline MetS 11 & 66 & $\mathrm{~m}$ & None & 32.1 & 29.1 & Insulin resistant \\
\hline MetS 12 & 63 & $\mathrm{~m}$ & Statin & 30.4 & 27.0 & Prediabetic \\
\hline MetS 13 & 69 & $\mathrm{~m}$ & Statin & 37.5 & 33.6 & Severe risk for insulin resistance \\
\hline MetS 14 & 76 & $\mathrm{~m}$ & None & 22.9 & 22.2 & Prediabetic \\
\hline MetS 15 & 79 & w & None & 26.9 & 25.5 & Insulin resistant \\
\hline MetS 16 & 60 & w & None & 32.7 & 28.8 & Insulin resistant \\
\hline MetS 17 & 56 & $\mathrm{~m}$ & Antidepressive & 32.8 & 28.1 & Prediabetic \\
\hline MetS 18 & 57 & $\mathrm{~m}$ & None & 31.4 & 25.4 & Insulin resistant \\
\hline MetS 19 & 49 & $\mathrm{~m}$ & None & 26.9 & 23.8 & Insulin resistant \\
\hline MetS 20 & 70 & $\mathrm{~m}$ & None & 38.8 & 34.2 & Insulin resistant \\
\hline MetS 21 & 43 & w & Statin & 36.9 & 32.0 & Insulin resistant \\
\hline Mean BMI & & & & 31.3 & 27.8 & \\
\hline
\end{tabular}

\section{Statistics}

A two-sample $t$-test for dependent samples (pairwise comparison test) was applied using Excel. Statistical significance was considered at $\mathrm{p}<0.05$.

\section{Results}

\section{Typical dietary pattern of MetS, prediabetes and Type 2 Diabetes patients observed}

The macromolecular dietary pattern of patients with MetS, prediabetes, or Type 2 Diabetes of both patient groups who had an initial TyG value higher than baseline shows a similar initial pathological nutritional pattern irrespective of BMI. An average intake of saturated fats of $35 \%$ and unsaturated fats of $46 \%$; an average glucose intake of $82 \mathrm{~g} / \mathrm{d}$, and an average fiber intake of $20 \mathrm{~g} / \mathrm{d}$ was observed.

\section{Type 2 Diabetes remission results}

The hepatic intermittent fasting therapy delivered efficient results, reversing Type 2 Diabetes within 60 days for all participants as measured by fasting glucose ( $\triangleright$ Fig. 1). Antidiabetic medications could be omitted for all patients at the latest at day 60 of the VLCD part of the therapy. The reprogrammed metabolism in these 60 days was stabilized during the personalized whole food nutrition part of the therapy up to 150 days, as shown in > Fig. 1. All patients could avoid their antidiabetic medications during the 90 days of the personalized whole food phase, and the diabetes remission rate was estimated to be $85 \%$, according to $\mathrm{HbA}_{1 \mathrm{c}}$ and fasting glucose levels.

\section{Adjuvant Type 2 Diabetes therapy results for uncontrolled diabetes}

- Table 4 shows results achieved in a patient with uncontrolled diabetes.

\section{MetS, NAFL, and prediabetes therapy results}

In the MetS group, 21 patients were insulin resistant according to the TyG index value, and six were prediabetic. The duration of the therapy needed to reprogram the metabolisms of all patients in the MetS group and get them stably out of the diseased path was up to 150 days ( $>$ Fig. 2 ). The method shows fast results within 30 to 50 days.

\section{Discussion and Conclusions}

\section{The paradox of satiety and nutrient hunger irrespective of BMI}

A similar macro- and micromolecular dietary pattern observed for MetS, prediabetes, or Type 2 Diabetes patients with too high intake 
a

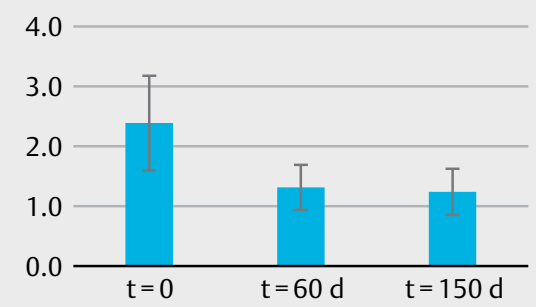

c

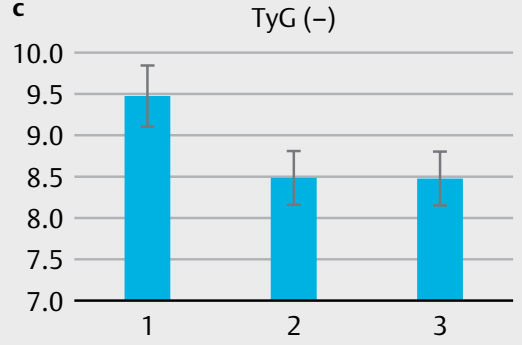

e

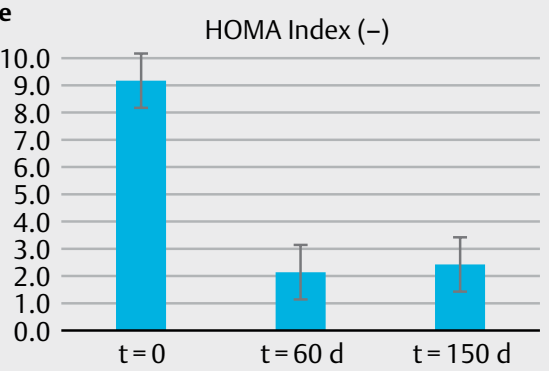

g

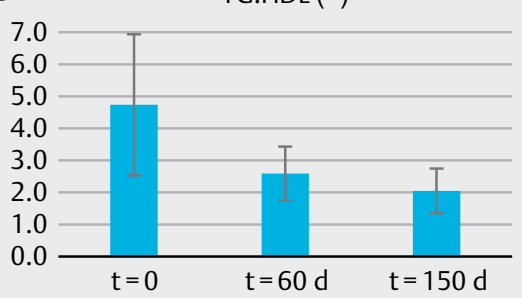

b

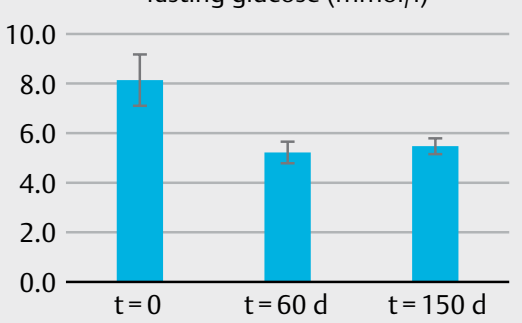

d

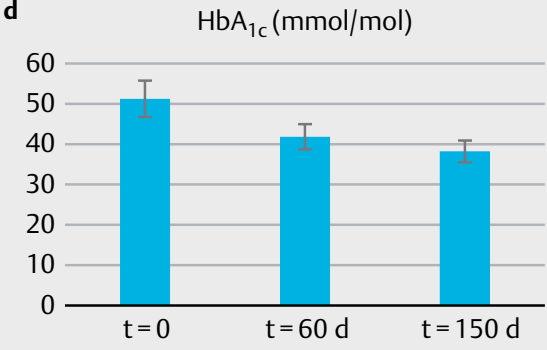

f

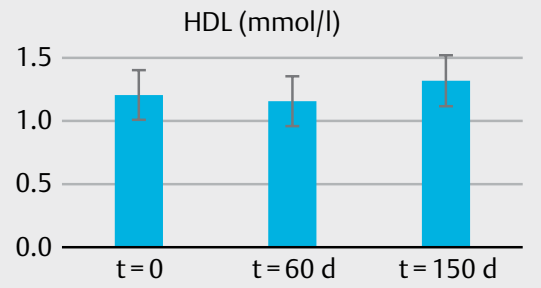

h

LDL (mmol/l)

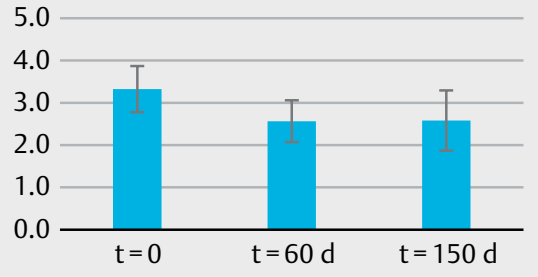

- Fig. 1 Median results of the diabetic remission group $(n=13)$ at three points in time: start $(t=0)$, after 60 days of VLCD, and after 150 days with the personalized whole food application. The single lead parameters and the corresponding surrogate biomarkers TyG and TG:HDL, as the leading medical indices for nutritional health, are shown. All data with $p<0.001$, confidence interval $95 \%$. a Triglycerides, threshold value $1.7 \mathrm{mmol} / \mathrm{l}$; b fasting glucose, threshold value $5.6 \mathrm{mmol} / \mathrm{l}$ for normal value, threshold value for diabetes remission $<7.0 \mathrm{mmol} / \mathrm{l}$; c TyG, calculated from a and b, threshold value $8.73 ; \mathbf{d ~} \mathrm{HbA}_{1 \mathrm{c}}$, threshold value for diabetes $<47.54 \mathrm{mmol} / \mathrm{mol} ; \mathbf{e} \mathrm{HOMA}$ Index, threshold value for Type 2 Diabetes $>3$; $\mathbf{f} \mathrm{HDL}$ values, threshold value $>1.0 \mathrm{mmol} / \mathrm{l} ; \mathbf{g}$ TG: $\mathrm{HDL}$ ratio, threshold value for insulin resistance $>3.0 ; \mathbf{h ~ L D L}$, threshold value $3.3 \mathrm{mmol} / \mathrm{l}$. $\mathrm{HbA}_{1 \mathrm{c}}$ and fasting glucose are the relevant Type 2 Diabetes remission parameters, as defined.

of saturated fats, unsaturated fats and glucose intake and a too low intake of fiber is in close agreement as reported by Breen et al. [50]. At the outset, all participants also deviated from the RDA of micronutrients magnesium, potassium, and vitamin $D$, as also reported by McKaye [63]. This may point to a new paradox for insulin-resistant risks, showing an abundance of glucose and palmitic acid intake, a shortage in unsaturated fat intake, and a scarcity for micronutrients, irrespective of BMI. No strong correlation with a typical macromolecular nor micromolecular dietary pattern could be extracted concerning the severity of the insulin resistance, nor BMI nor waist circumference. But a molecular nutrition deviation irre- spective of BMI leading to insulin resistance is particular congruent with nutritional stress for hypertriglyceridemia, hyperinsulinemia and low HDL-cholesterol. As a consequence a combination of TyG and TG:HDL as lead biomarkers for leading the food intake to structure the personalized program for optimal diabetes remission was chosen.

\section{Type 2 Diabetes remission is not driven by calories only}

TyG as the lead surrogate biomarker for nutritional feeding allows structuring personalized whole food nutrition for diabetes remis- 
sion for all BMI categories ( $\geq 25$ ), and a nutritional structure that is not just focused on calories (as is the main approach in today's marketplace). Caloric aspects are taken into account of the personalization algorithm of the diet, but calories are not the leading factor only and are calculated according to the body's energy needs. For Patient 8 ( $\vee$ Table 1 ) this is shown in detail ( $\vee$ Table 3 ). Patient 8 presented a starting BMI of 26.8. The patient's diet before therapy, with a high intake of saturated fats and glucose, and low fiber intake with a typical in-between snacking behavior, showed similar calorie intake (isocaloric) and macronutrient distribution to her diet after the therapy ( $\triangleright$ Table 3 ).

- Table $3 \mathrm{BMI}$ is not the driver for Type 2 Diabetes remission. Isocaloric nutrition of a patient before and at the end of therapy, no medication, <1-year diagnosis, female, 47 years, initial BMI 26.8.

\begin{tabular}{|c|c|c|}
\hline $\begin{array}{l}\text { Isocaloric nutrition, } \\
\text { composition }\end{array}$ & Before therapy & After therapy \\
\hline Total calories kcal/day & 1676 & 1560 \\
\hline Carbohydrate $\mathrm{g} / \mathrm{d}$ & 188 & 160 \\
\hline Carbohydrate kcal/d & 771 & 656 \\
\hline $\begin{array}{l}\text { \% carbohydrate of total } \\
\text { calorie intake }\end{array}$ & 53 & 54 \\
\hline Proteins g/d & 80 & 83 \\
\hline Proteins as $\mathrm{kcal} / \mathrm{d}$ & 330 & 338 \\
\hline $\begin{array}{l}\% \text { protein of total calorie } \\
\text { intake }\end{array}$ & 20 & 22 \\
\hline Total fat $\mathrm{g} / \mathrm{d}$ & 66 & 63 \\
\hline Fat as kcal/d & 611 & 590 \\
\hline$\%$ of total fat intake & 36 & 38 \\
\hline Saturated fat $\mathrm{g} / \mathrm{d}$ & 27.6 & 12.5 \\
\hline Monounsaturated fat $\mathrm{g} / \mathrm{d}$ & 21.6 & 28.2 \\
\hline Polyunsaturated fat $\mathrm{g} / \mathrm{d}$ & 8.6 & 17.6 \\
\hline Omega-3 fatty acids g/d & 0.8 & 3.3 \\
\hline Fiber $\mathrm{g} / \mathrm{d}$ & 26.7 & 44.9 \\
\hline $\begin{array}{l}\% \text { fiber of total calorie } \\
\text { intake }\end{array}$ & 6.5 & 11.8 \\
\hline Vitamin D $\mu \mathrm{g} /$ day & 1.3 & 9.2 \\
\hline Magnesium mg/day & 279.0 & 577.0 \\
\hline Potassium g/d & 2.4 & 4.3 \\
\hline $\begin{array}{l}\text { Fasting blood values| } \\
\text { anthropometric data }\end{array}$ & Before therapy & After therapy \\
\hline Triglyceride mmol/l & 2.7 & 1.4 \\
\hline $\mathrm{HDL} \mathrm{mmol/I}$ & 1.2 & 1.3 \\
\hline LDL mmol/l & 3.9 & 3.1 \\
\hline Glucose $\mathrm{mmol} / \mathrm{l}$ & 9.1 & 5.4 \\
\hline $\mathrm{HbA}_{1 \mathrm{c}} \mathrm{mmol} / \mathrm{mol}$ & 50.8 & 38.8 \\
\hline HOMA & 8.5 & 2.7 \\
\hline TyG & 9.9 & 8.7 \\
\hline TG:HDL & 5.1 & 2.4 \\
\hline BMI kg/m² & 26.8 & 22.6 \\
\hline Waist circumference $\mathrm{cm}$ & 97.0 & 90.0 \\
\hline
\end{tabular}

As can be seen, the molecular distribution of the fatty acid fraction was completely changed after therapy. After therapy, saturated fat intake values were normal $(<13 \mathrm{~g})$; intake of fiber was above the recommended value of $40 \mathrm{~g} / \mathrm{d}$; and vitamin $D$, magnesium, and potassium all reached values above the RDA. The HOMA index continued to improve after the end of the therapy (after 150 days) and was estimated to be 1.57 after 323 days.

\section{Personalized molecular nutrition for Type 2 Diabetes remission}

A highly efficient and effective insulin resistance remission result was achieved for all patients across all BMI categories, according to the lead biomarker: $85 \%$ of the patients (11 out of 13) achieved remission, and only one patient did not reach the lower threshold value of the TG:HDL ratio of 3.0. The TG:HDL ratio combined with TyG are the leading biomarkers but are weighted more to stabilizing the metabolism reprogramming that occurs during the VLCD part of the therapy. Our finding confirmed Achilike et al. [75], who found that TG:HDL has the power to produce a metabolically healthy phenotype. The personalized nutritional strategy reduces the triglyceride levels, and the molecular personalized whole food diet reduces the buildup of new triglycerides, leading to a negative balance that reaches normal levels over time. It is of utmost importance to keep the triglyceride buildup under control since triglycerides can cross the blood-brain barrier and contribute to decreased satiety, as reported by Banks et al. [76].

Improvements in triglycerides were very fast during VLCD, averaging $48 \%$. The fasting glucose adaptation during VLCD was also very fast, and all patients reached the threshold value $<7 \mathrm{mmol} / \mathrm{I}$ within the first 14 days.

Ten patients reached fasting glucose values $<5.6 \mathrm{mmol} / \mathrm{l}$, and all patients were better than $6.3 \mathrm{mmol} / \mathrm{l}$ after completing the therapy (150 days). On average, fasting glucose values improved by $33 \%(2.66 \mathrm{mmol} / \mathrm{l})$.

Diabetes remission was always accompanied by weight loss as a symptomatic effect of the metabolic improvements. Kelly et al. [77] reported a mean diabetes remission rate of $49.4 \%$ applying very low calorie diets. The VLCD part of the intermittent hepatic therapy, which is focused on the cause of insulin resistance, delivers more effective results, as our reported data show ( $\triangleright$ Fig. 1).

The weight loss reached was not homogenous, since the initial $\mathrm{BMI}$ was not, and the therapy approach is not focused on calories only. The statistical significance and regression coefficient for diabetes reversion against the HOMA Index was highest for the TyG Index $(0.61, \mathrm{p}<0.001)$, compared to the TG:HDL Index $(0.41$, $\mathrm{p}<0.05)$. The weight loss achieved also showed a lower regression correlation to the HOMA Index $(0.43, p<0.05)$. This implies that the TyG index is the key biomarker for diabetes remission, with a strong focus on triglyceride normalization as a key success factor. This does reflect the current research that triglycerides are driving hyperinsulinemic conditions that disturb the glucose metabolism, leading to weight increase as a homeostatic defending mechanism.

The HOMA Index as a medically accepted analytical value for insulin resistance was always surpassed, and on average was reduced by $79 \%$, or 6.7 units over the entire therapy length. For one hyperinsulinemia patient, an improvement in the HOMA Index of 12 times was observed. All patients reached the $<3$ threshold point of 
- Table 4 Time course of a Type 2 Diabetes patient with uncontrolled diabetes (male, 55 years).

\begin{tabular}{|c|c|c|c|c|}
\hline Analysis & Before & One month after & Norm value & Unit \\
\hline Cholesterol & $2.74^{*}$ & $2.4^{*}$ & $<5.2$ & $\mathrm{mmol} / \mathrm{l}$ \\
\hline HDL cholesterol & 0.77 & 0.79 & $>1.0$ & $\mathrm{mmol} / \mathrm{l}$ \\
\hline LDL cholesterol & $1.14^{*}$ & $1.21^{*}$ & $<3.3$ & $\mathrm{mmol} / \mathrm{l}$ \\
\hline Triglycerides & $3.58^{*}$ & $1.55^{*}$ & $<1.7$ & $\mathrm{mmol} / \mathrm{l}$ \\
\hline Glucose & $12.28^{* *}$ & $5.77^{* *}$ & $3.9-5.6$ & $\mathrm{mmol} / \mathrm{l}$ \\
\hline TyG & 10.45 & 8.86 & $<8.73$ & - \\
\hline ASAT/GOT & 0.62 & 0.63 & $<0.85$ & $\mu \mathrm{mol} / \mathrm{s}^{*} \mathrm{I}$ \\
\hline ALAT/GPT & 0.88 & 0.69 & $<0.85$ & $\mu \mathrm{mol} / \mathrm{s}^{*} \mathrm{I}$ \\
\hline GGT & 1.24 & 1.05 & $<1.19$ & $\mu \mathrm{mol} / \mathrm{s}^{*} \mathrm{I}$ \\
\hline Cortisol & 56 & $<28$ & & $\mathrm{nmol} / \mathrm{l}$ \\
\hline Insulin (ip) & 0.52 & 0.09 & $0.02-0.12$ & $\mathrm{nmol} / \mathrm{l}$ \\
\hline HOMA Index & $39.2^{* *}$ & $3.2^{* *}$ & $<3$ & - \\
\hline Waist circumference & 115 & 109 & $<94$ & $\mathrm{~cm}$ \\
\hline BMI & 31.6 & 29.4 & $20-25$ & $\mathrm{~kg} / \mathrm{m}^{2}$ \\
\hline Bodyweight & 99.8 & 92.9 & & $\mathrm{~kg}$ \\
\hline
\end{tabular}

the HOMA Index (3.8 reached for this patient, with a starting HOMA Index of 28). Over the full course of the therapy, a success rate of $>90 \%$ could be estimated according to the HOMA Index. All patients of the diabetes group reached $\mathrm{HbA}_{1 \mathrm{c}}$ values $<47.54 \mathrm{mmol} /$ $\mathrm{mol}$, at the end of the 150 days time window. The average improvement in $\mathrm{HbA}_{1 \mathrm{c}}$ was $17 \%$, or $21.8 \mathrm{mmol} / \mathrm{ml}$, which is higher than the $6.65 \mathrm{mmol} / \mathrm{mol}$ achieved in the caloric diabetes remission study reported by Ades et al. [78] that had a VLCD with a similar therapy duration of six months.

The importance of the triglyceride metabolism as presented is further supported by Ma et al. [79] and Lim et al. [80] who report that triglyceride levels are independently correlated with insulin resistance and islet beta-cell function in individuals with dyslipidemia.

We observed a relationship between the time required to achieve the threshold value of fasting glucose and the prior duration of Type 2 Diabetes and medication taken, but due to the limited number of patients this observation is in conclusive.

\section{Adjuvant diabetes therapy to the uncontrolled medicated patient}

Uncontrolled diabetes can be improved efficiently by restoring normal triglyceride levels, leading to normal insulin levels, by applying the personalized whole food part of the therapy ( $\triangleright$ Table 4 ).

The initial fasting glucose value of $12.28 \mathrm{mmol} / \mathrm{I}$ was reduced to $5.7 \mathrm{mmol} / \mathrm{l}$ in 30 days, while keeping the antidiabetic medication unchanged. Insulin resistance according to the HOMA Index was reduced by a factor of more than 10 (from 39.2 to 3.2), almost reaching the lower threshold value of 3.0 within 30 days of application. The TyG index estimated was 8.86 after application (threshold 8.73) and indicates improved status or almost no remaining in- sulin resistance. The triglycerides level was reduced from $3.58 \mathrm{mmol} / \mathrm{l}$ to $1.55 \mathrm{mmol} / \mathrm{l}$ (but still taking statins). Cortisol was reduced from 56 to $<28$. The vicious cycle of increased cortisol paired with insulin resistance leading to hyperinsulinemia driving visceral fat was changed from a pathologic insulin level from 0.52 to a normal insulin level of 0.09 within 30 days of application.

Triglycerides have emerged as a new risk factor for cardiovascular disease in Type 2 Diabetes, as reported by Ye et al. [81]. According to Halldin et al. [82], the risk factor cholesterol has shifted more toward the risk factor triglycerides. Statins do not lower triglycerides adequately, as reported by Toth et al. [83] and, as reported by Shimoda et al. [84], the durability of the glucose-lowering effects from DPP-4 inhibitors can be better maintained if strict triglyceride management takes place.

\section{Insulin resistance remission for MetS and prediabetes patients normalizing triglycerides}

Normalization of MetS, prediabetes, and amelioration of NAFL is mediated by the reduction of triglycerides, similar to what is observed in Type 2 diabetes patients. The patients reported in their anamnesis a weight loss resistance which can be overcome with a fast normalization of the dyslipidemic state and triglyceride levels within the two first weeks of the personalized whole food part of the therapy. The molecular nutrition focused on the "one carbon" and CPT1 metabolism of the whole food part of the intermittent hepatic therapy delivers fast triglyceride amelioration. After 30 days of application of the personalized whole food intermittent fasting therapy part, the average TyG index was improved by $9 \%$. Sixteen patients reached the lower threshold value $<8.73$ concerning insulin resistance but also TG: $\mathrm{HDL}<3$. This corresponds to $>76 \%$ efficiency for insulin resistance normalization respectively remission. 

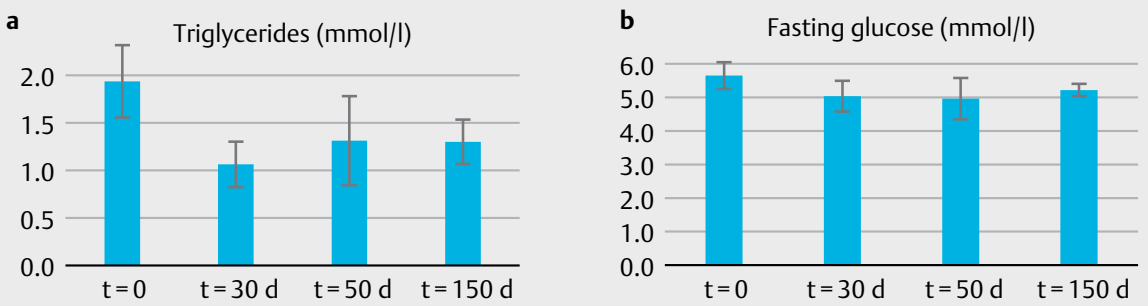

C

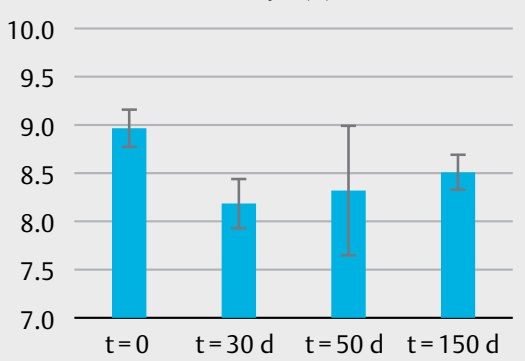

e

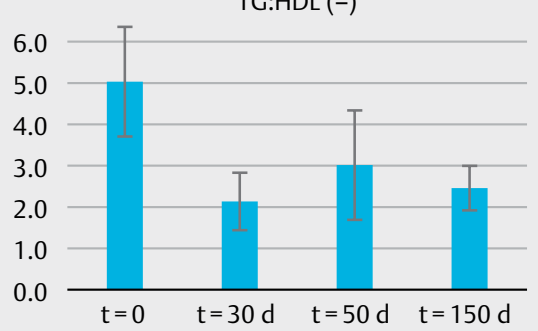

d

$\mathrm{HDL}(\mathrm{mmol} / \mathrm{l})$

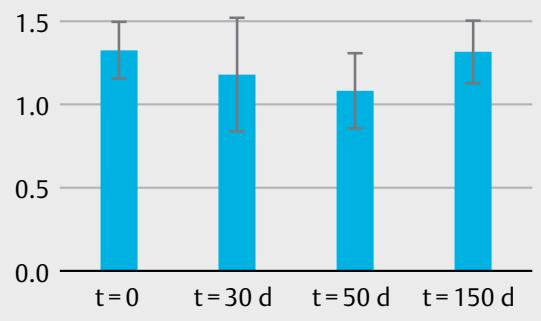

f

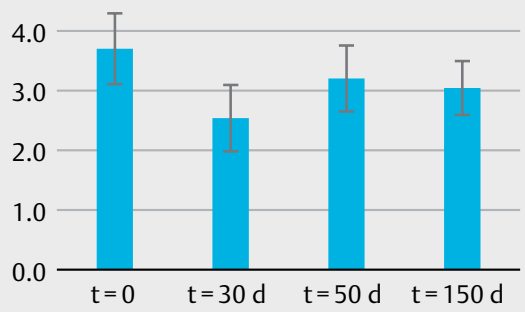

- Fig. 2 Median results of the MetS/prediabetic group $(n=21)$ at four-time windows: start $t=0$, at 30 days, at 50 days, and at 150 days. The single lead parameters and the corresponding surrogate biomarkers TyG and TG:HDL ratio as the leading medical index for nutritional health, are shown. All data with $\mathrm{p}<0.001$, confidence interval $95 \%$ a Triglyceride course, threshold value $1.7 \mathrm{mmol} / \mathrm{l}$; $\mathbf{b}$ fasting glucose course, threshold value $5.6 \mathrm{mmol} / \mathrm{l}$ for normal; $\mathbf{c}$ TyG course, calculated from $\mathbf{a}$ and $\mathbf{b}$, threshold value 8.73 ; $\mathbf{d}$ HDL values, threshold value >1.0 mmol/l; e TG:HDL ratio, threshold value for insulin resistance > $3.0 ; \mathbf{f L L}$, threshold value $3.3 \mathrm{mmol} / \mathrm{l}$.

Those five patients who were still slightly above the threshold showed good improvement but needed more time to reach the lower threshold. All initially prediabetic patients were no longer prediabetic after 150 days of application. The average improvement in triglyceride reduction was $33 \%$ within 150 days. This is in accordance to Farell etal. [85] of key importance in breaking weight loss resistance, since early and established NAFL is responsible for this effect due to triglyceride accumulation. We measured an average improvement of the liver index within the first 50 days of 29 units. The initial median liver index was estimated as 81 units. According to Khan etal. [86], the modulation of triglycerides and insulin resistance represents a potential new strategy for NAFL treatment.

Triglyceride values stabilized after 50 days, HDL cholesterol increased, and LDL cholesterol decreased, stabilizing the metabolic and weight loss goals that had been reached.

Weight reduction occurred as a result of the metabolic improvement, with a mean $\mathrm{BMI}$ reduction of about $8 \%$ within 50 days and up to $11 \%$ within 150 days. To date, weight loss is the only existing therapy for NAFL according to Hydes et al. [87]. Our results suggest that, with a triglyceride and insulin resistance focus paired for weight loss, a new efficient NAFL treatment option is available.
As conclusion, the applied therapeutically method allows reversing the Type 2 Diabetic metabolism within 60 days and remission within 150 days with high efficiency. This shifts the treatment paradigm for Type 2 Diabetes from management to cure. Applying these method to patients with MetS and/or prediabetes can normalize their insulin resistance or prediabetes completely within 50 to 150 days. Our results favor a molecular nutritional diabetes prevention and remission strategy that is focused on triglyceride normalization with TyG as the lead biomarker, but that also combines a caloric approach enabling dietary molecular nutrition with an epigenetic point of view as a completely new diabetes prevention and remission strategy, and also a new treatment option for NAFL patients.

The pilot study has strengths and also weaknesses. Its major strength is the achievement of insulin resistance and Type 2 Diabetes remission in a significant, fast, and highly efficient way. The method is completely digitally supported for easy application and combines smart formulas of active ingredients to support the nutritional and metabolic adaptation. The presented method can establish a new treatment option that cures Type 2 Diabetes. The weakness of the pilot study is a limited number of persons and inhomogeneous patient characteristics. 
Additional studies might be of interest from a scientific point of view, both to measure further data from more homogenous patient groups, and also to explore the epigenetic effects involved. However, from a practical and an application point of view, the methodology is very robust, proven, simply to apply, completely digitally supported, and ready to be used on a larger scale. Applying this new paradigm of a personalized molecular dietary pattern control for insulin resistance remission could revolutionize diabetes medicine and could also contribute beneficially to reduce the economic burden of Type 2 Diabetes and its related diseases.

\section{Conflict of Interest}

The authors declare that they have no conflict of interest.

\section{References}

[1] Madsen KS, Chi Y, Metzendorf MI et al. Metformin for prevention or delay of type 2 diabetes mellitus and its associated complications in persons at increased risk for the development of type 2 diabetes mellitus. Cochrane Database System Rev 2019; 1-122

[2] Ferrannini E, Gall W, Nannipieri M et al. Identification of novel insulin resistance metabolites in a non-diabetic population by global biochemical profiling. Canad J Diabetes 2009; 33: 208

[3] Nannini RD, Joyce BT, Zheng $Y$ et al. Epigenetic age acceleration and metabolic syndrome in the coronary artery risk development in young adults study. Clin Epigenet 2019; 160-169

[4] De Rosa V, Galgani M, Santopaolo M et al. Nutritional control of immunity: Balancing the metabolic requirements with an appropriate immune function. Semin Immunol 2015; 27: 300-309

[5] Chavez-Reyes ], Escarcega-Gonzalez CE, Chavira-Suarez E et al. Susceptibility for Some Infectious Diseases in Patients with Diabetes: The Key Role of Glycemia. Front Public Health 2021; 9: 559-595

[6] Vestergaard Jensen A, Faurholt-Jepsen D, Banbaek Egelund G et al. Undiagnosed Diabetes Mellitus in Community-Acquired Pneumonia: A Prospective Cohort Study. Clin Infect Dis 2017; 29: 2091-2098

[7] Gardner JP, Li S, Srinavasan SR et al. Rise in Insulin Resistance Is Associated with Escalated Telomere Attrition. Circulation 2005; 111: 2171-2177

[8] Chen S, Chen Y, Liu X et al. Association of Insulin Resistance with Chronic Kidney Disease in Non-Diabetic Subjects with Normal Weight. PLoS One 2013; 1-9

[9] Won Lee, Ha Park S. Association between depression and nonalcoholic fatty liver disease: Contributions of insulin resistance and inflammation. J Affect Disord 2021; 278: 259-263

[10] Geraets AF], Köhler S, Muzambi R et al. The association of hyperglycemia and insulin resistance with incident depressive symptoms over 4 years of follow-up: The Maastricht Study. Diabetologia 2020; 63: 2315-2328

[11] Rundek T, Gardener H, Xu Q et al. Insulin resistance and risk of ischemic stroke among nondiabetic individuals from the northern Manhattan study. Arch Neurol 2010; 67: 1195-1200

[12] Ford AH, Flicker L, Graeme G] et al. Insulin resistance and depressive symptoms in older men: the health in men study. Am J Geriatr Psychiatry 2015; 23: 872-880

[13] Nägga K, Gustavsson AM, Stomrud E et al. Increased midlife triglycerides predict brain $\beta$-amyloid and tau pathology 20 years later. Neurology 2018; 90: e73-e81
[14] Sung H, Siegel RL, Rosenberg PS et al. Emerging cancer trends among young adults in the USA: Analysis of a population-based cancer registry. Lancet 2019; 4: E137-E147

[15] Li X, Li G, Cheng T et al. Association between triglyceride-glucose index and risk of incident diabetes: A secondary analysis based on a Chinese cohort study: TyG index and incident diabetes. Lipids Health Dis 2020; 8: 236

[16] Ren $\mathrm{H}$, Yang Y, Wang $\mathrm{F}$ et al. Association of the insulin resistance marker TyG index with the severity and mortality of COVID-19. Cardiovasc Diabetol 2020; 19: 58

[17] Corona G, Pittocaro A, Vena W et al. Diabetes is the most important cause for mortality in COVID-19 hospitalized patients: Systematic review and meta-analysis. Rev Endocr Metab Disord 2021; 1-22

[18] McGovern AP, Thomas NJ, Vollmer S] et al. The disproportionate excess mortality of COVID-19 in younger people with diabetes warrants vaccination prioritization. Diabetologia 2021; 64: 1184-1186

[19] Kaiser A, Vollenweider P, Waeber $G$ et al. Prevalence, awareness and treatment of type 2 diabetes mellitus in Switzerland: the CoLaus study. Diabet Med 2012; 29: 190-197

[20] Araujo J, Cai J, Stevens J. Prevalence of Optimal Metabolic Health in American Adults: National Health and Nutrition Examination Survey 2009-2016. Metab Syndr Relat Disord 2019; 17: 46-52

[21] Kerr D, Miles P. Very low-calorie diets in diabetes: The Bournemouth experience. J Diabetes Nursing 2000; 4: 108-111

[22] Willi SM, Martin K, Datko FM et al. Treatment of Type 2 Diabetes in Childhood Using a Very-Low-Calorie Diet. Diabetes Care 2004; 27: 348-353

[23] Xin Y, Davies A, Briggs A et al. Type 2 diabetes remission: 2-year within-trial and lifetime-horizon cost-effectiveness of the Diabetes Remission Clinical Trial (DiRECT)/Counterweight-Plus weight management program. Diabetologia 2020; 63: 2112-2122

[24] Baskota A, Li S, Dhakal N et al. Bariatric Surgery for Type 2 Diabetes Mellitus in Patients with BMI $<30 \mathrm{~kg} / \mathrm{m} 2$ : A Systematic Review and Meta-Analysis. PLoS One 2015; 10: e0132335

[25] Vistisen D, Witte DR, Tabak AG et al. Patterns of Obesity Development before the Diagnosis of Type 2 Diabetes: The Whitehall II Cohort Study. PLoS Med 2014; 11: e1001602

[26] Wu S, Fischer-Hoch SP, Reninger B et al. Metabolic Health Has Greater Impact on Diabetes than Simple Overweight/Obesity in Mexican Americans. J Diabetes Res 2016; Article ID 4094876

[27] Eckel N, Li Y, Kuxhaus O et al. Transition from metabolic healthy to unhealthy phenotypes and association with cardiovascular disease risk across BMI categories in 90257 women (the Nurses' Health Study): 30-year follow-up from a prospective cohort study. Lancet Diabetes Endocrinol 2018; 6: 714-724

[28] Pietiläinen KH, Sysi-Aho M, Rissanen A et al. Acquired obesity is associated with changes in the serum lipidomic profile independent $\mathrm{pf}$ genetic effects - a monozygotic twin study. PLoS One 2007; 2: e218

[29] Owei I, Umekwe N, Provo C et al. Insulin-sensitive and insulin-resistant obese and non-obese phenotypes: Role in the prediction of incident pre-diabetes in a longitudinal biracial cohort. BMJ Open Diabetes Res Care 2017; 5: e000415

[30] Mongraw-Chaffin M, Foster MC, Anderson CAM et al. Metabolically healthy obesity, transition to metabolic syndrome, and cardiovascular risk. J Am Coll Cardiol 2018; 71: 1857-1865

[31] Bagnatti M, Ogunkolade W, Marshall C et al. Glucolipotoxicity initiates pancreatic $\beta$-cell death through TNFR5/CD40-mediated STAT1 and NF-KB activation. Cell Death Disease 2016; 7: 2329

[32] Telles S, Pal S, Sharma SK et al. The association between the lipid profile and fasting blood glucose with weight-related outcomes in healthy obese adults. BMC Res Notes 2018; 11: 383 
[33] Lee J, Kim B, Kim W et al. Lipid indices as simple and clinically useful surrogate markers for insulin resistance in the U.S. population. Sci Rep 2021; 11: 2366

[34] Lim TK, Lee HS, Lee Y]. Triglyceride to HDL-cholesterol ratio and the incidence risk of type 2 diabetes in community-dwelling adults: A longitudinal 12-year analysis of the Korean Genome and Epidemiology Study. Diabetes Res Clin Pract 2020; 163: 108150

[35] Rojas-Humpire R, Olarte-Durand M, Medina-Ramirez S et al. Insulin Resistance Indexes as Biomarkers of Lifetime Cardiovascular Risk among Adults from Peru. J Nutr Metab 2021; 6633700: 1-8

[36] Si Y, Fan W, Shan W et al. Association between triglyceride glucose index and coronary artery disease with type 2 diabetes mellitus in middle-aged and elderly people. Medicine (Baltimore) 2021; 100: e25025

[37] Arpon A, Milagro FI, Santos JL et al. Interaction among sex, aging, and epigenetic process concerning visceral fat, insulin resistance, and dyslipidemia. Front Endocrinol 2019; 10: 496

[38] Çin Aslan NN, Yardimci HH, Koç N et al. Triglycerides/high-density lipoprotein cholesterol is a predictor similar to the triglyceride-glucose index for the diagnosis of metabolic syndrome using International Diabetes Federation criteria of insulin resistance in obese adolescents: a cross-sectional study. J Pediatr Endocrinol Metab 2020; 33: 777-784

[39] Kron V, Verner M, Smetana P et al. The Changes of Cholesterol Profile at the Different Insulin Resistance Range in the Czech Republic. Medicina (Kaunas) 2021; 57: 249

[40] Lyu K, Zhang Y, Zhang D et al. A Membrane-Bound Diacylglycerol Species Induces PKCE-Mediated Hepatic Insulin Resistance. Cell Metab 2020; 32: 654-664

[41] Lee Y, Lai HTM, de Oliveira Otto MC et al. Serial biomarkers of de novo lipogenesis fatty acids and incident heart failure in older adults: The Cardiovascular Health Study. J Am Heart Assoc 2020; 9: e014119

[42] Rasmussen BB, Holmbäck UF, Volpi E et al. Malonyl coenzyme A and the regulation of functional carnitine palmitoyltransferase- 1 activity and fat oxidation in human skeletal muscle. J Clin Invest 2002; 110: 1687-1693

[43] Imamura F, Fretts AM, Marklund $M$ et al. Fatty acids in the de novo lipogenesis pathway and incidence of type 2 diabetes: A pooled analysis of prospective cohort studies. PLoS Med 2020; 17: e1003102

[44] Kitae A, Hashimoto Y, Hamaguchi M et al. The triglyceride and glucose index is a predictor of incident nonalcoholic fatty liver disease: A Population-Based Cohort Study. Can J Gastroenterol Hepatol 2019; 121574: $1-7$

[45] Jimenez-Rivera C, Hadjiyannakis S, Davila J et al. Prevalence and a risk factor for non-alcoholic fatty liver in children and youth with obesity. BMC Pediatrics 2017; 17: 113

[46] Kolb H, Kempf K, Röhling M et al. Insulin: too much of a good thing is bad. BMC Med 2020; 18: 224

[47] Wadolowska L, Hamulka J, Kowalkowska J et al. Skipping Breakfast and a Meal at School: Its Correlates in Adiposity Context. Report from the ABC of Healthy Eating Study of Polish Teenagers. Nutrients 2019; 11 : 1563

[48] Joo HJ, Kim GR, Park EC et al. Association between Frequency of Breakfast Consumption and Insulin Resistance Using Triglyceride-Glucose Index: A Cross-Sectional Study of the Korea National Health and Nutrition Examination Survey (2016-2018). Int J Environ Res Public Health 2020; 17: 3322

[49] Jakubowicz D, Landau S, Tsameret S et al. Reduction in Glycated Hemoglobin and Daily Insulin Dose Alongside Circadian Clock Upregulation in Patients with Type 2 Diabetes Consuming a ThreeMeal Diet: A Randomized Clinical Trial. Diabetes Care 2019; 42: 2171-2180

[50] Breen C, Ryan M, McNulty B et al. High saturated-fat and low-fiber intake: A comparative analysis of nutrient intake in individuals with and without type 2 diabetes. Nutr Diabetes 2014; 4: e104
[51] Tiedemann L], Schmid SM, Hettel ] et al. Central insulin modulates food valuation via mesolimbic pathways. Nat Commun 2017; 8: 16052

[52] Pugnaloni S, Alia SMancini et al. A Study on the Relationship between Type 2 Diabetes and Taste Function in Patients with Good Glycemic control. Nutrients 2020; 12: 1112-1122

[53] Luukkonen PK, Sädevirta S, Zhou Y et al. Saturated Fat Is More Metabolically Harmful to the Human Liver Than Unsaturated Fat or Simple Sugars. Diabetes Care 2018; 41: 1732-1739

[54] Fryk E, Olausson J, Mossberg K et al. Hyperinsulinemia and insulin resistance in the obese may develop as part of a homeostatic response to elevated free fatty acids: A mechanistic case-control and a population-based cohort study. Lancet 2021; 65: 103264

[55] Grubelnik V, Zmazek JMarkovič et al. Mitochondrial Dysfunction in Pancreatic Alpha and Beta-cells Associated with Type 2 Diabetes Mellitus. Life 2020; 10: 348

[56] Hannon BA, Thompson SV, Edwards CG et al. Dietary Fiber Is Independently Related to Blood Triglycerides Among Adults with Overweight and Obesity. Curr Dev Nutr 2018; 28: 094

[57] Dong Y, Chen L, Gutin B et al. Total, Insoluble, and Soluble Dietary Fiber Intake and Insulin Resistance and Blood Pressure in Adolescents. Eur J Clin Nutr 2019; 73: 1172-1178

[58] Gao X, Wang Y, Sun G. High dietary choline and betaine intake is associated with low insulin resistance in the Newfoundland population-. Nutrition 2017; 33: 28-34

[59] Azemati B, Rajaram S, Jaceldo-Siegl K et al. Animal-Protein Intake Is Associated with Insulin Resistance in Adventist Health Study 2 (AHS-2) Calibration Substudy Participants: A Cross-Sectional Analysis. Curr Dev Nutr 2017; 1: e000299

[60] Meir AY, Rinott E, Tsaban G et al. Effect of green-Mediterranean diet on intrahepatic fat: the DIRECT PLUS randomized controlled trial. Gut. 2021; 1-11

[61] Castro-Barquero SA Tresserra-Rimbau A, Vitelli-Storelli F et al. Dietary Polyphenol Intake is Associated with HDL-Cholesterol and A Better Profile of Other Components of the Metabolic Syndrome: A PREDIMED-Plus Sub-Study. Nutrients 2020; 12: 689-696

[62] McKaye J, Ho S, Jane M et al. Overweight \& obese Australian adults and micronutrient deficiency. BMC Nutr 2020; 1: 12

[63] Hall E, Volkov P, Daveh T et al. Effects of palmitate on genome-wide mRNA expression and DNA methylation patterns in human pancreatic islets. BMC Med 2014: 12: 103

[64] Maples ], Brault JJ, Wiczak CA et al. Differential epigenetic and transcriptional response of the skeletal muscle carnitine palmitoyltransferase 1B (CPT1B) gene to lipid exposure with obesity. Am J Physiol Endocrinol Metab 2015; 309: E345-E356

[65] Bacha F, Klinepeter Bartz S, Puvau M et al. Metabolic flexibility across the spectrum of glycemic regulation in youth. JCI Insight 2021; 6: e146000

[66] Irvin MR, Joehanes R, Mendelson M et al. Epigenome-wide association study of fasting blood lipids in the Genetics of Lipid-lowering Drugs and Diet Network study. Circulation 2014; 130: 565-572

[67] Mamtani M, Kulkarni H, Dyer TD et al. Genome- and epigenome-wide association study of the hypertriglyceridemic waist in Mexican American families. Clin Epigenetics 2016; 6: 1-14

[68] Lai C, Parnell LD, Smith CE et al. Carbohydrate and fat intake associated with risk of metabolic diseases through epigenetics of CPT1A. Am J Clin Nutr 2020; 112: 1200-1211

[69] Arpón A, Milagro FI, Ramos-Lopez O et al. Epigenome-wide association study in peripheral white blood cells involving insulin resistance. Sci Rep 2019; 9: 2445-2456

[70] Rohner M. Method for evaluating foods, and nutritional systems for the prevention and treatment of chronic disease. European Patent EP3166639B1. 
[71] Rohner M. Method for Weight Reduction. European Patent EP1962826B1.

[72] Simental-Mendía LE, Rodríguez-Morán M, Guerrero-Romero F. The product of fasting glucose and triglycerides as a surrogate for identifying insulin resistance in apparently healthy subjects. Metab Syndr Relat Disord 2008; 6: 299-304

[73] Masson W, Siniawski D, Lobo M et al. Association between triglyceride/ HDL cholesterol ratio and carotid atherosclerosis in postmenopausal middle-aged women. Endocrinol Nutr 2016; 63: 327-332

[74] Bedogni G, Bellentani S, Miglioli L et al. The Fatty Liver Index: a simple and accurate predictor of hepatic steatosis in the general population. BMC Gastroenterol 2006; 6: 33

[75] Achilike I, Hazuda HP, Fowler SP et al. Predicting the development of the metabolically healthy obese phenotype. Int J Obes 2015; 39 : 228-234

[76] Banks WA, Farr SA, Salameh TS et al. Triglycerides cross the bloodbrain barrier and induce central leptin and insulin receptor resistance. Int ] Obes 2018; 42: 391-397

[77] Kelly ], Karlsen M, Steinke G. Type 2 Diabetes Remission and Lifestyle Medicine: A Position Statement from the American College of Lifestyle Medicine.. Am J Lifestyle Med 2020; 14: 406-419

[78] Ades PA, Savage PD, Marney AM et al. Remission of recently diagnosed type 2 diabetes mellitus with weight loss and exercise. J Cardiopulm Rehabil Prev 2015; 35: 193-197

[79] Ma M, Liu H, Yo J et al. Triglyceride is independently correlated with insulin resistance and islet beta-cell function: a study in a population with different glucose and lipid metabolism states. Lipids Health Disease 2020; 19: 121-133
[80] Lim EL, Hollingsworth KG, Aribisala M] et al. Reversal of type 2 diabetes: normalization of beta-cell function in association with decreased pancreas and liver triacylglycerol. Diabetologia 2011; 54: 2506-2514

[81] Ye X, Kong W, Zafar Ml et al. Serum triglycerides as a risk factor for cardiovascular diseases in type 2 diabetes mellitus: a systematic review and meta-analysis of prospective studies. Cardiovasc Diabetol 2019; 18: $48-58$

[82] Halldin AK, Björkelund C. Cholesterol and triglyceride levels in midlife and risk of heart failure in women, a longitudinal study: the prospective population study of women in Gothenburg. BMJ Open 2020; 10: 1-8

[83] Toth PP, Philip S, Hull M et al. Association of Elevated Triglycerides with Increased Cardiovascular Risk and Direct Costs in Statin-Treated Patients. Mayo Clin Proc 2019; 94: 1670-1680

[84] Shimoda M, Miyoshi-Takai M, Irie S et al. Inadequate Triglyceride Management Worsens the Durability of Dipeptidyl Peptidase-4 Inhibitor in Subjects with Type 2 Diabetes Mellitus. J Diabetes Res 2017; 5856475: 1-8

[85] Khan RS, Bril F, Cusi K et al. Modulation of Insulin Resistance in Nonalcoholic Fatty Liver Disease. Hepatology 2018; 70: 711-724

[86] Farell A, Green H, Williams J et al. Adapting medical weight loss strategies to NAFL - is it effective). AASLD 2019; 065

[87] Hydes T], Favi S, Loomba R et al. Evidence-based clinical advice for nutrition and dietary weight loss strategies for the management of NAFL and NASH. Clin Mol Hepatol 2020; 26: 383-400 